\title{
Calculation of Feynman diagrams using a symbolic manipulation programme in personal computers
}

\author{
W.G.D. Dharmaratna ${ }^{*}$ and M.A.A. Karunarathna \\ Department of Physics, University of Ruhuna. Matara. Sri Lanka
}

\begin{abstract}
A method to calculate Feynman diagrams is developed using a symbolic manipulation package that runs in a personal computer. Commercially available package called "Mathematica" is used. The method to calculate scattering amplitudes in the lowest order in the perturbation theory is demonstrated by explicitly calculating a Feynman diagram in Quantum Chromodyanamics. The procedure described here can be easily extended to calculate any Feynman diagram including next to leading order diagrams and also to the calculation of Feynman diagrams in Quantum electrodyanamics.
\end{abstract}

\section{INTRODUCTION}

Interactions of elementary particles are well understood in relativistic quantum field theories (QFT) ${ }^{1}$. However, the calculation of observable quantities in QFT is a very lengthy and a time consuming process. One method of calculation of scattering amplitudes is the use of perturbation theory, which is well established. The rules for the calculation of scattering amplitudes are conveniently illustrated with Feynman diagrams $^{1,2}$. One can calculate Feynman diagrams without the help of computers but it is time consuming, especially, in calculating higher order diagrams. However, after the development of symbolic manipulation programme called MACSYMA, the calculation of Feynman diagrams has been performed efficiently using main frame computers such as $\operatorname{VAX}^{3,4}$.

\footnotetext{
* Corresponding author
} 
With the availability of fast personal computers and symbolic manipulation packages for PCs, it is quite useful to develop procedures to calculate Feynman diagrams in PCs, especially, if one doesn't have access to expensive computers. We have developed a method to calculate Feynman diagrams using the symbolic manipulation package 'MATHEMATICA' ${ }^{5}$. This paper describes, in detail, how to utilize the package in calculating scattering amplitudes in Quantum Chromodynamics ${ }^{2}$ (i.e. the QFT of strong interaction, which describes the interaction of quarks and gluons).

For a given hadronic interaction, there are a large number of partonic subprocesses that could contribute to observable quantities. The aim here is to illustrate the essential steps on how one could use Mathematica to calculate Feynman diagrams and not to demonstrate calculations of all sub-processes, which have been already calculated by other means. Therefore, only one subprocess, namely, the gluon fusion subprocess (gluon +gluon $\rightarrow$ quark + anti-quark) is considered here. This subprocess involves all essential components such as Dirac spinors for quarks and anti-quarks, $\gamma$ matrices, polarization vectors for gluons etc. The extension of the procedure to other subprocesses is straightforward.

\section{METHOD OF CALCULATION OF AMPLITUDES}

The first step is to define kinematics, the Dirac spinors for quarks and antiquarks, polarization vectors for gluons and scattering amplitudes. In using the symbolic manipulation package it is convenient to define amplitudes in the helicity basis. Then these quantities have to be incorporated into Mathematica.

\subsection{Definition of Helicity Amplitudes}

For a two body scattering process $\mathrm{A}+\mathrm{B} \rightarrow \mathrm{C}+\mathrm{D}$ with each particle having two spin states (as for spin $1 / 2$ fermions or massless spin 1 particles), there are 16 different helicity amplitudes. Parity invariance of the strong interaction reduces the number of independent helicity amplitudes to 8 . For the gluon fusion process, the invariance under charge conjugaton and the identical particle interchange reduces the number of independent amplitudes to 6 . The six independent helicity amplitudes can be chosen as ${ }^{6}$

$$
\begin{aligned}
& \phi 1(++\rightarrow++) \\
& \phi 2(++\rightarrow--) \\
& \phi 3(+-\rightarrow+-) \\
& \phi 4(+-\rightarrow-+) \\
& \phi 5(++\rightarrow-+) \\
& \phi 6(+-\rightarrow++)
\end{aligned}
$$


according to the specific quark helicity states $(\mathrm{ab} \rightarrow \mathrm{cd}$ ) in the initial and final states, where a, b, c, \& d, which takes + or -, are the helicities of particle A, B, C, \& D, respectively.

\subsection{Kinematics}

The calculation of helicity flip amplitudes is performed in the center of mass frame of the sub process. The system of coordinate axes that is used in the calculation is shown in Figure 1. The axes are chosen in such a way that the scattering plane is normal to the $\mathrm{y}$-axis and the momentum of particle A defining the direction of $\mathrm{z}$-axis. Fourmomenta of particles A, B, C, \& D are labeled p1, p2, p3 \& p4 respectively. The angle between the particle $\mathrm{C}$ and the $\mathrm{z}$-axis is called $\theta$. The kinematics has to be defined separately for each sub process since particles involved possess different masses. Also, spinors for quarks and anti-quarks and polarization vectors for gluons have to be defined for each process. They are defined in the helicity basis and are given in following sections for the gluon fusion process.
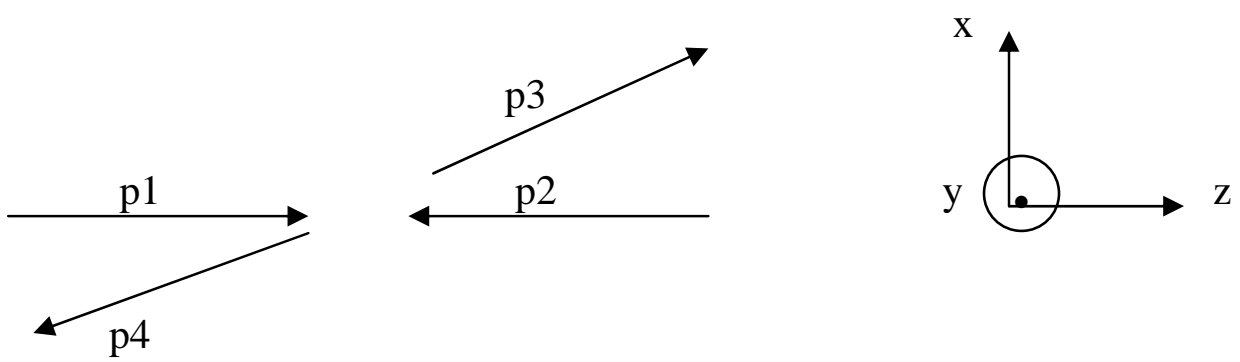

Fig 1. System of coordinate axes used.

\subsection{Spinors and Polarization vectors}

For the gluon fusion sub-process, the produced quark and anti-quark are chosen to be particles C \& D, respectively, while the initial state gluons represent A \& B. Fourmomenta of particles for this sub process in the above coordinate system can be written as

$$
\begin{array}{ll}
\mathrm{p} 1 \equiv(\mathrm{p}, 0,0, \mathrm{p}) & \mathrm{p} 2 \equiv(\mathrm{p}, 0,0,-\mathrm{p}) \\
\mathrm{p} 3 \equiv(\mathrm{e}, \mathrm{k} \sin \theta, 0, \mathrm{k} \cos \theta) & \mathrm{p} 4 \equiv(\mathrm{e}, \mathrm{k} \sin \theta, 0,-\mathrm{k} \cos \theta)
\end{array}
$$


where $\mathrm{p}$ is the center-of-mass momentum of gluons in the initial state, which is different from that of quarks ( $\mathrm{k}$ ) in the final state, and e is the energy of the quark (or anti-quark). The conservation of energy gives $\mathrm{e}=\mathrm{p}$. With this kinematics, the Dirac spinors for quark $\left(\mathrm{u}_{ \pm}\right)$and anti-quark $\left(\mathrm{v}_{ \pm}\right)$take the following form,

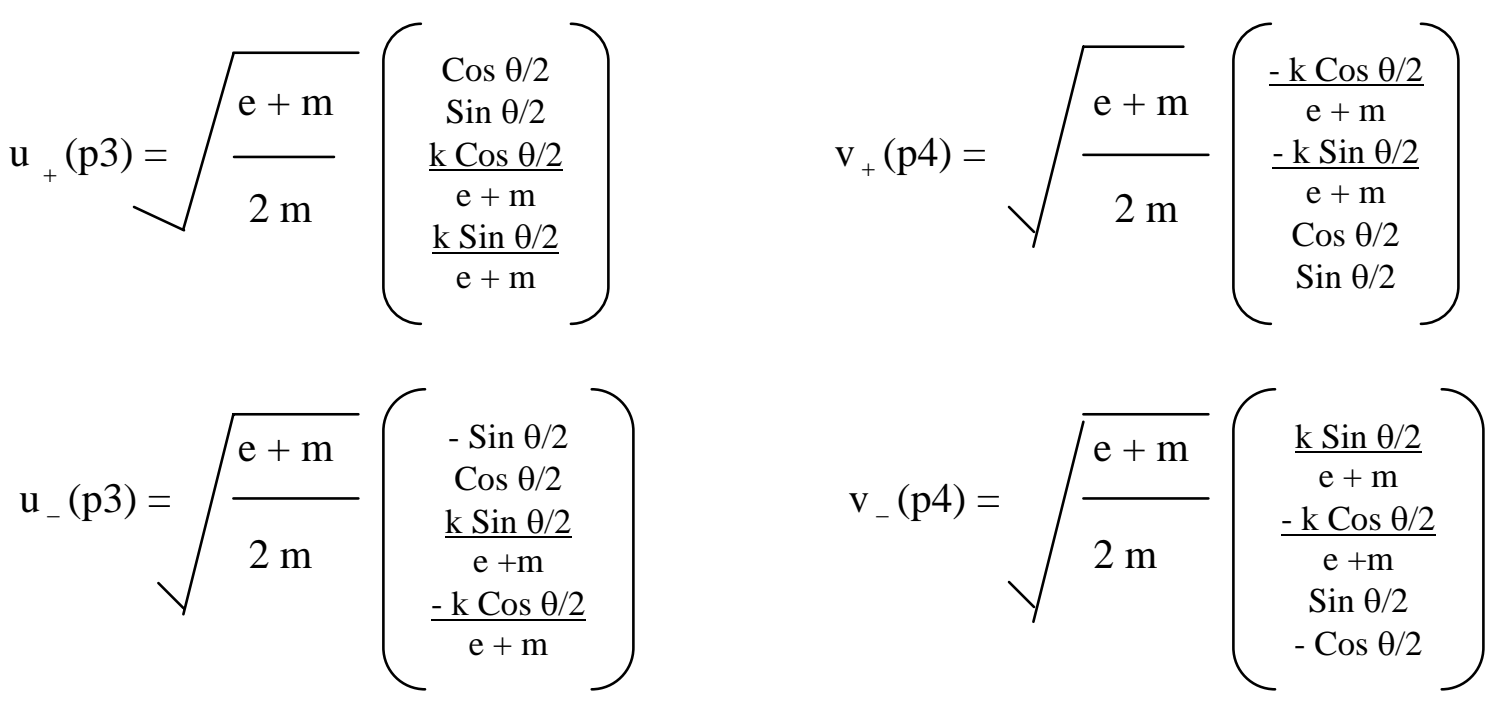

where, $\mathrm{m}$ is the mass of the quark or anti-quark in the final state. The polarization vectors in the initial state gluons take the form

$$
\begin{array}{ll}
\varepsilon_{+}^{1} \equiv \frac{1}{\sqrt{2}}(0,-1,-i, 0) & \varepsilon_{+}^{2} \equiv \frac{1}{\sqrt{2}}(0,1,-i, 0) \\
\varepsilon_{-}^{1} \equiv \frac{1}{\sqrt{2}}(0,1,-i, 0) & \varepsilon_{-}^{2} \equiv \frac{1}{\sqrt{2}}(0,-1,-i, 0) .
\end{array}
$$

\subsection{Scattering amplitudes}

There are three Feynman diagrams that contribute to the $g+g \rightarrow q+\bar{q}$ subprocess in the lowest order as shown in Figure 2. The lowest order $M$-matrix can be obtained by calculating these three diagrams. The relevant notations for colour (i,j...a,b...), momenta and polarization vectors are indicated. Neglecting the colour factors and the Figure 2. iii), which includes the three gluon coupling, one can use the same procedure to calculate analogues Quantum Electrodynamics sub-process. 
i)

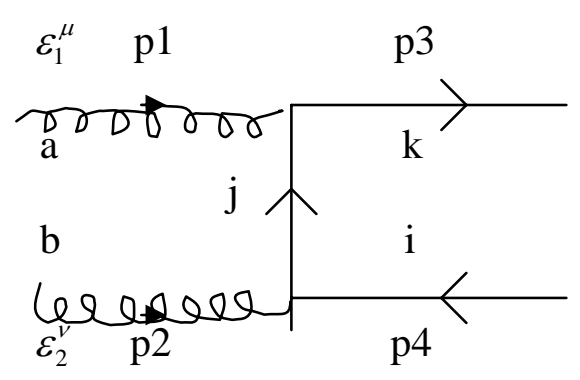

ii)

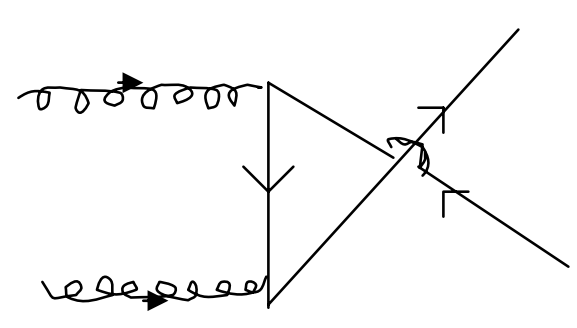

iii)

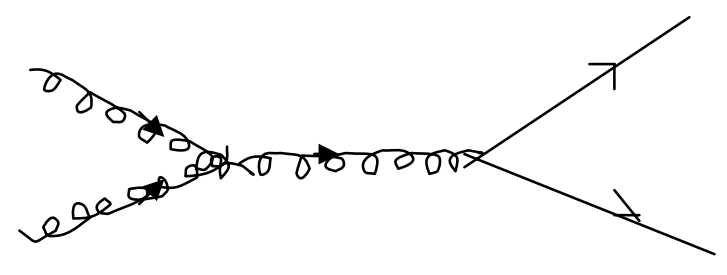

Fig 2. Lowest order Feynman diagrams for gluon fusion.

It is straightforward to write the amplitudes for the three diagrams using Feynman rules ${ }^{2}$. The sum of contributions from the three Feynman diagrams, the lowest order $M$-matrix, can be expressed as follows.

$$
\begin{aligned}
& M=-g^{2}\left(\lambda^{c} \lambda^{b}\right)_{i k}\left[\frac{\bar{u}\left(p_{3}\right)\left[2 p_{3} \cdot \varepsilon_{1}-\phi_{1} p_{1}\right]-\phi_{2} v\left(p_{4}\right)}{2 p_{1} \cdot p_{3}}+\left(\bar{u}\left(p_{3}\right) \frac{\gamma^{\kappa}}{\hat{s}} v\left(p_{4}\right)\right) C_{\mu v \kappa} \varepsilon_{1}^{\mu} \varepsilon_{2}^{v}\right] \\
& -g^{2}\left(\lambda^{b} \lambda^{c}\right)_{i k}\left[\frac{\bar{u}\left(p_{3}\right) \phi_{2}\left[p_{1} \phi_{1}-2 p_{4} \cdot \varepsilon_{1}\right]-v\left(p_{4}\right)}{-2 p_{1} \cdot p_{4}}-\left(\bar{u}\left(p_{3}\right) \frac{\gamma^{\kappa}}{\hat{s}} v\left(p_{4}\right)\right) C_{\mu v \kappa} \varepsilon_{1}^{\mu} \varepsilon_{2}^{v}\right]
\end{aligned}
$$

where

$$
C_{\mu \nu \kappa}=g_{\mu \nu}\left(p_{1}-p_{2}\right)_{\kappa}+g_{v \kappa}\left(2 p_{2}+p_{1}\right)_{\mu}+g_{\kappa \mu}\left(-2 p_{1}-p_{2}\right)_{v}
$$

and the notation $\phi=\gamma_{\mu} a^{\mu}$ is used. Note that the relation between the colour factors if $f_{b c a} \lambda_{i k}^{a}=\left[\lambda_{b}, \lambda_{c}\right]_{i k}$ is used in the simplification. Finally, the helicity amplitudes can be written in the form

$$
\phi_{\alpha}=-g^{2}\left[\left(\lambda^{c} \lambda^{b}\right)_{i k} f_{\alpha}+\left(\lambda^{b} \lambda^{c}\right)_{i k} h_{\alpha} .\right.
$$




\section{INCORPORION INTO MATHEMATICA}

Having defined the amplitudes, the next step is to incorporate all quantities into the Mathematica package. This section describes how one can define all necessary quantities in Mathematica.

\subsection{Four-momenta of particles and $\gamma$ matrices}

Four-momenta ( $\left.p^{\mu}\right)$ of particles (section 2.C) can be defined as four-dimensional arrays. First declare the array and then define each component.

Array[p1,4], p1[1]=p, p1[2]=0, p1[3]=0, p1[4]=p

Array[p2,4], p2[1]=p, p2[2]=0, p2[3]=0, p2[4]=-p

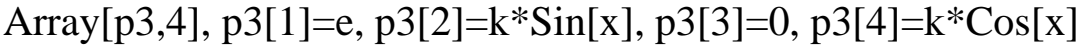

Array[p4,4], p4[1] =e, p4[2]=-k*Sin[x], p4[3] $=0, p 4[4]=-k * \operatorname{Cos}[x]$

Similarly, $\gamma^{\mu}$ can be define as a four-dimensional array, but the components are now matrices.

Array[Gam,4];

$\operatorname{Gam}[1]=\{\{1,0,0,0\},\{0,1,0,0\},\{0,0,-1,0\},\{0,0,0,-1\}\}$

$\operatorname{Gam}[2]=\{\{0,0,0,1\},\{0,0,1,0\},\{0,-1,0,0\},\{-1,0,0,0\}\}$

$\operatorname{Gam}[3]=\{\{0,0,0,-\mathrm{I}\},\{0,0, \mathrm{I}, 0\},\{0, \mathrm{I}, 0,0\},\{-\mathrm{I}, 0,0,0\}\}$

$\operatorname{Gam}[4]=\{\{0,0,1,0\},\{0,0,0,-1\},\{-1,0,0,0\},\{0,1,0,0\}\}$

Note that in the standard formulation the $\gamma$ matrices are called $\gamma^{0}, \gamma^{1}, \gamma^{2}$ and $\gamma^{3}$. Here, they are labeled as $\gamma^{1}, \gamma^{2}, \gamma^{3}$ and $\gamma^{4}$, respectively, in order to define them in an array in Mathematica.

\subsection{Dirac Spinors and Polarization Vectors}

Dirac spinors defined in section 2.C can be incorporated into Mathematica as one-dimensional arrays of two components. The calculation of amplitude needs the helicity states of $\bar{u}\left(p_{3}\right)$ (the product of the transpose of $u\left(p_{3}\right)$ and $\gamma^{1}$ ) for the quark states. $\bar{u}_{+}\left(p_{3}\right), \bar{u}_{-}\left(p_{3}\right), v_{+}\left(p_{4}\right)$ and $v_{-}\left(p_{4}\right)$ are defined as u3[1], u3[2], v4[1] and $\mathrm{v} 4[2]$, respectively, as given below.

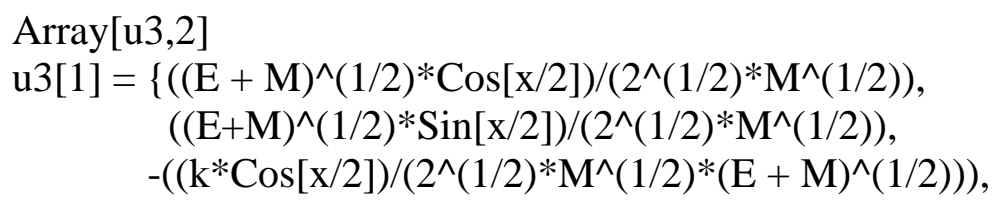




$$
\begin{aligned}
& \left.-\left(\left(\mathrm{k}^{*} \operatorname{Sin}[\mathrm{x} / 2]\right) /\left(2^{\wedge}(1 / 2)^{*} \mathrm{M}^{\wedge}(1 / 2)^{*}(\mathrm{E}+\mathrm{M})^{\wedge}(1 / 2)\right)\right)\right\} \\
\mathrm{u} 3[2]= & -\left(\left((\mathrm{E}+\mathrm{M})^{\wedge}(1 / 2)^{*} \operatorname{Sin}[\mathrm{x} / 2]\right) /\left(2^{\wedge}(1 / 2)^{*} \mathrm{M}^{\wedge}(1 / 2)\right)\right), \\
& \left((\mathrm{E}+\mathrm{M})^{\wedge}(1 / 2)^{*} \operatorname{Cos}[\mathrm{x} / 2]\right) /\left(2^{\wedge}(1 / 2)^{*} \mathrm{M}^{\wedge}(1 / 2)\right), \\
& -\left(\left(\mathrm{k}^{*} \operatorname{Sin}[\mathrm{x} / 2]\right) /\left(2^{\wedge}(1 / 2)^{*} \mathrm{M}^{\wedge}(1 / 2)^{*}(\mathrm{E}+\mathrm{M})^{\wedge}(1 / 2)\right)\right), \\
& \left.\left(\mathrm{k}^{*} \operatorname{Cos}[\mathrm{x} / 2]\right) /\left(2^{\wedge}(1 / 2)^{*} \mathrm{M}^{\wedge}(1 / 2)^{*}(\mathrm{E}+\mathrm{M})^{\wedge}(1 / 2)\right)\right\}
\end{aligned}
$$

$$
\begin{aligned}
\text { Array[}[ & \mathrm{v} 4,2] \\
\mathrm{v} 4[1]=\{ & -\left(\left(\mathrm{k}^{*} \operatorname{Cos}[\mathrm{x} / 2]\right) /\left(2^{\wedge}(1 / 2)^{*} \mathrm{M}^{\wedge}(1 / 2)^{*}(\mathrm{E}+\mathrm{M})^{\wedge}(1 / 2)\right)\right), \\
& -\left(\left(\mathrm{k}^{*} \operatorname{Sin}[\mathrm{x} / 2]\right) /\left(2^{\wedge}(1 / 2)^{*} \mathrm{M}^{\wedge}(1 / 2)^{*}(\mathrm{E}+\mathrm{M})^{\wedge}(1 / 2)\right)\right), \\
& \left((\mathrm{E}+\mathrm{M})^{\wedge}(1 / 2)^{*} \operatorname{Cos}[\mathrm{x} / 2]\right) /\left(2^{\wedge}(1 / 2)^{*} \mathrm{M}^{\wedge}(1 / 2)\right), \\
& \left.\left((\mathrm{E}+\mathrm{M})^{\wedge}(1 / 2)^{*} \operatorname{Sin}[\mathrm{x} / 2]\right) /\left(2^{\wedge}(1 / 2)^{*} \mathrm{M}^{\wedge}(1 / 2)\right)\right\} \\
\mathrm{v} 4[2]= & \left(\left(\mathrm{k}^{*} \operatorname{Sin}[\mathrm{x} / 2]\right) /\left(2^{\wedge}(1 / 2)^{*} \mathrm{M}^{\wedge}(1 / 2)^{*}(\mathrm{E}+\mathrm{M})^{\wedge}(1 / 2)\right),\right. \\
& -\left(\left(\mathrm{k}^{*} \operatorname{Cos}[\mathrm{x} / 2]\right) /\left(2^{\wedge}(1 / 2)^{*} \mathrm{M}^{\wedge}(1 / 2)^{*}(\mathrm{E}+\mathrm{M})^{\wedge}(1 / 2)\right)\right), \\
& \left((\mathrm{E}+\mathrm{M})^{\wedge}(1 / 2)^{*} \operatorname{Sin}[\mathrm{x} / 2]\right) /\left(2^{\wedge}(1 / 2)^{*} \mathrm{M}^{\wedge}(1 / 2)\right), \\
& \left.-\left(\left((\mathrm{E}+\mathrm{M})^{\wedge}(1 / 2)^{*} \operatorname{Cos}[\mathrm{x} / 2]\right) /\left(2^{\wedge}(1 / 2)^{*} \mathrm{M}^{\wedge}(1 / 2)\right)\right)\right\}
\end{aligned}
$$

Note that $\mathrm{u} 3[1]$ and $\mathrm{u3}[2]$ are defined as row matrices and $\mathrm{v} 4[1]$ and $\mathrm{v} 4[2]$ are defined as column matrices. The helicity + and - states are labeled by 1 and 2, respectively. The angle $\theta$ is labeled as $\mathrm{x}$ in Mathematica.

Polarization vectors can be introduced into the Mathematica as two-dimensional arrays. The first dimension has two components, 1 and 2, to represent helicity states + and -, respectively, and the second dimension has four components to represent the four components of the polarization vector.

Array[E1, $\{2,4\}]$;

$$
\begin{array}{r}
\mathrm{E} 1[1,1]=0, \mathrm{E} 1[1,2]=-2 \wedge(-1 / 2), \mathrm{E} 1[1,3]=-\mathrm{I} / 2^{\wedge}(1 / 2), \mathrm{E} 1[1,4]=0 \\
\mathrm{E} 1[2,1]=0, \mathrm{E} 1[2,2]=2^{\wedge}(-1 / 2), \mathrm{E} 1[2,3]=-\mathrm{I} / 2^{\wedge}(1 / 2), \mathrm{E} 1[2,4]=0 \\
\operatorname{Array}[\mathrm{E} 2,\{2,4\}] ;
\end{array}
$$

\subsection{Amplitudes}

In order to define the amplitudes in Mathematica, two more quantities, the metric tensor, $g_{\mu v}$, and the slash vectors, $\phi=\gamma_{\mu} a^{\mu}$, are needed. One-dimensional array of four components, $\mathrm{G}$ as defined below, can be used to handle the metric tensor, since it is a diagonal matrix.

$$
\text { Array }[G, 4] ; G[1]=1, G[2]=-1, G[3]=-1, G[4]=-1
$$

With this definition, $p_{1}$ can be defined as 


$$
\text { Slp1 := Sum[G[m]*Gam[m]*p1[m], \{m, 1, 4\}]. }
$$

Note that the contraction of four-vectors are performed using the Mathematica command Sum, which takes the summation over indices 1 to 4 . The amplitudes $f_{\alpha}$ and $h_{\alpha}$ in the helicity basis depend on the helicity states of the four particles involved. Therefore, the amplitudes can be defined as four-dimensional arrays, each dimension having two components for two helicity states. They are defined below.

$\mathrm{f}\left[\mathrm{i}_{-}, \mathrm{j}_{-}, \mathrm{r}_{-}, \mathrm{l}_{-}\right]:=$ u3[r].Sum[(2*p3[m]*Gam[n] - Gam[m].Slp1.Gam[n])*G[m]*E1[i, m]*G[n]*E2[j, n], $\{\mathrm{m}, 1,4\},\{\mathrm{n}, 1,4\}] . \mathrm{v} 4[\mathrm{l}] /\left(-2 * \mathrm{E}^{*} \mathrm{p}+2 * \mathrm{k}^{*} \mathrm{p} * \operatorname{Cos}[\mathrm{x}]\right)+\mathrm{u} 3[\mathrm{r}] .(\operatorname{Sum}[\mathrm{Gam}[\mathrm{x}] * \mathrm{G}[\mathrm{x}] *$ (p1[x] - p2[x]), \{x, 1, 4\}]* Sum[E1[i, m]*G[m]*E2[j, m], \{m, 1, 4\}]) . v4[l]/(4*p^2) + $\mathrm{u} 3[\mathrm{r}] \cdot(\operatorname{Sum}[(2 * \mathrm{p} 2[\mathrm{~m}]+\mathrm{p} 1[\mathrm{~m}]) * \mathrm{G}[\mathrm{m}] * \mathrm{E} 1[\mathrm{i}, \mathrm{m}],\{\mathrm{m}, 1,4\}] * \operatorname{Sum}[\mathrm{Gam}[\mathrm{x}] * \mathrm{G}[\mathrm{x}] * \mathrm{E} 2[\mathrm{j}$, $\mathrm{x}],\{\mathrm{x}, 1,4\}]) . \mathrm{v} 4[1] /\left(4 * \mathrm{p}^{\wedge 2}\right)+\mathrm{u} 3[\mathrm{r}] .(\operatorname{Sum}[(-2 * \mathrm{p} 1[\mathrm{n}]-\mathrm{p} 2[\mathrm{n}]) * \mathrm{G}[\mathrm{n}] * \mathrm{E} 2[\mathrm{j}, \mathrm{n}], \quad\{\mathrm{n}, 1,4\}]$ *Sum[Gam $[\mathrm{x}] * \mathrm{G}[\mathrm{x}] * \mathrm{E} 1[\mathrm{i}, \mathrm{x}],\{\mathrm{x}, 1,4\}])$. v4[1]/(4*p^2)

$\mathrm{h}\left[\mathrm{i}_{-}, \mathrm{j}_{-}, \mathrm{r}_{-}, \mathrm{l}_{-}\right]:=$ u3[r] . Sum[(-2*p4[m]*Gam[n] + Gam[n] . Slp1 . Gam[m] $* G[m] * E 1[i, m] * G[n] *$ E2 $[j$, $\mathrm{n}],\{\mathrm{m}, 1,4\},\{\mathrm{n}, 1,4\}] . \mathrm{v} 4[1] /\left(-2 * \mathrm{E}^{*} \mathrm{p}-2 * \mathrm{k}^{*} \mathrm{p} * \operatorname{Cos}[\mathrm{x}]\right)-\mathrm{u} 3[\mathrm{r}] .\left(\operatorname{Sum}\left[\mathrm{Gam}[\mathrm{x}]^{*} \mathrm{G}[\mathrm{x}]\right.\right.$ *(p1[x] - p2[x]), \{x, 1, 4\}]*Sum[E1[i, m]*G[m]*E2[j, m], \{m, 1, 4\}]) . v4[1]/ (4*p^2) + u3[r] .(Sum[(2*p2[m] + p1[m])*G[m]*E1[i,m], \{m, 1, 4\}]*Sum[Gam[x] * G[x]*E2[j, $\mathrm{x}],\{\mathrm{x}, 1,4\}]) \cdot \mathrm{v} 4[1] /\left(4^{*} \mathrm{p}^{\wedge} 2\right)+\mathrm{u} 3[\mathrm{r}] .\left(\operatorname{Sum}\left[\left(-2^{*} \mathrm{p} 1[\mathrm{n}]-\mathrm{p} 2[\mathrm{n}]\right)^{*} \mathrm{G}[\mathrm{n}] * \mathrm{E} 2[\mathrm{j}, \mathrm{n}],\{\mathrm{n}, 1\right.\right.$, $4\}] * \operatorname{Sum}[\operatorname{Gam}[\mathrm{x}] * \mathrm{G}[\mathrm{x}] * \mathrm{E} 1[\mathrm{i}, \mathrm{x}],\{\mathrm{x}, 1,4\}]) \cdot \mathrm{v} 4[1] /\left(4 * \mathrm{p}^{\wedge} 2\right)$

Here i, j, k and l can take values 1 (for helicity +) or 2 (for helicity -). Note that the dots in above equations denote the matrix multiplication. After defining the amplitudes in the above form, the evaluation of the six independent helicity amplitudes is straightforward. The helicity amplitude $f_{1}(++\rightarrow++)$, for example, can be obtained by evaluating $\mathrm{f}[1,1,1,1]$. After the evaluation, the simplification of kinematics has to be performed. This can be done by using the Mathematica command Simplify. We can define commands to apply again and again as needed. For example, we can define a command, say, Simpk as

$$
\text { Simpk[x_] := Simplify[x /. } \left.\mathrm{k}^{\wedge} 2->\mathrm{p}^{\wedge} 2-\mathrm{M}^{\wedge} 2\right]
$$

to replace $k^{2}$ with $p^{2}-m^{2}$ in any expression $\mathrm{x}$ and to simplify the result. For the gluon fusion subprocess we obtained following results, which are in agreement with known results. 


$$
\begin{aligned}
& f_{1}=m(k+p) /\left(p^{2}-k p \operatorname{Cos}(x)\right) \\
& f_{2}=m(k-p) /\left(p^{2}-k p \operatorname{Cos}(x)\right) \\
& f_{3}=4 k \operatorname{Cos}^{3}\left(\frac{x}{2}\right) \operatorname{Sin}\left(\frac{x}{2}\right) /(p-k \operatorname{Cos}(x)) \\
& f_{4}=4 k \operatorname{Cos}\left(\frac{x}{2}\right) \operatorname{Sin}^{3}\left(\frac{x}{2}\right) /(p-k \operatorname{Cos}(x)) \\
& f_{5}=0 \\
& f_{6}=-m k \operatorname{Sin}^{2}(x) /\left(p^{2}-k p \operatorname{Cos}(x)\right)
\end{aligned}
$$

$$
\begin{aligned}
& h_{1}=m(k+p) /\left(p^{2}+k p \operatorname{Cos}(x)\right) \\
& h_{2}=m(k-p) /\left(p^{2}+k p \operatorname{Cos}(x)\right) \\
& h_{3}=4 k \operatorname{Cos}^{3}\left(\frac{x}{2}\right) \operatorname{Sin}\left(\frac{x}{2}\right) /(p+k \operatorname{Cos}(x)) \\
& h_{4}=4 k \operatorname{Cos}\left(\frac{x}{2}\right) \operatorname{Sin}^{3}\left(\frac{x}{2}\right) /(p+k \operatorname{Cos}(x)) \\
& h_{5}=0 \\
& h_{6}=-m k \operatorname{Sin}^{2}(x) /\left(p^{2}+k p \operatorname{Cos}(x)\right)
\end{aligned}
$$

\section{CONCLUSIONS AND DISCUSSION}

We have been successful in using the symbolic manipulation package, Mathematica, to perform the lowest order Feynman diagrams in Quantum Chromodynamics. The results obtained here are in agreement with known results. The method described here in the calculation of gluon fusion subprocess illustrates how one could use the package in calculating any Feynman diagram in the lowest order. With these illustrations of how Dirac matrices, spinors, polarization vectors and amplitudes are defined in Mathematica, it is a straightforward exercise to extend the calculation to other lowest order Feynman diagrams.

We have set up a programme to calculate other subprocesses such as, quark-quark scattering, quark-gluon scattering and quark annihilation process. Interested parties can obtain a copy of this programme by contacting the authors, if needed. It is interesting to note that the calculation of Feynman diagrams is very fast after the amplitudes are defined. As an example, once the amplitudes are defined, the calculation and simplification of six helicity amplitudes (lowest order) takes only about two minutes in a Pentium II computer when no other processes are running.

The calculation of next to leading order diagrams ${ }^{3,4}$ involves integration over the fourmomenta. Once the integration is completed, then the rest of the calculations can be performed in the same manner described here. Fourth order diagrams, certainly, would take a longer time. However, once the amplitudes are defined as above the interacting time can be minimized by running Mathematica in a batch mode. 
W.G.D. Dharmaratna. and M.A.A.. Karunarathna,/Sri Lankan Journal of Physics, Vol.1,(2000) 89-98

\section{REFERENCES}

1. C. Itzykson and J.B. Zuber, Quantum Field Theory, (McGraw-Hill, USA, 1980).

2. E. Leader and E. Predazzi, An Introduction to Gauge Theories and the New Physics, (Cambridge Univ. Press, Cambridge, 1980)

3. W. G. D. Dharmaratna and G. R. Goldstein, Gluon Fusion as a Source for Masive-quark Polarizaton, Phys. Rev. D, 41, 1731 (1990)

4. W. G. D. Dharmaratna and G. R. Goldstein, Single Particle Polarization in QCD Subprocesses, Phys. Rev. D, $\underline{53}, 1073(1996)$

5. S. Wolfram, Mathematica, $2^{\text {nd }}$ Edition (Addision-Wesley, 1991)

6. N. S. Craigie et. al., Spin Physics at Short Distances, Phys. Reports, 99, 69 (1983) 